NISTIR 7925

\title{
Method for Measuring Axis Orthogonality in MEMS Accelerometers
}

Craig D. McGray

Yaqub Afridi

Jon Geist 
NISTIR 7925

\title{
Method for Measuring Axis Orthogonality in MEMS Accelerometers
}

\author{
Craig D. McGray \\ Yaqub Afridi \\ Jon Geist \\ Semiconductor and Dimensional Division \\ Physical Measurement Laboratory
}

http://dx.doi.org/10.6028/NIST.IR.7925

July 2013

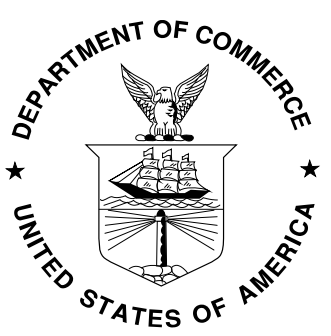

U.S. Department of Commerce

Penny Pritzker, Secretary

National Institute of Standards and Technology Patrick D. Gallagher, Under Secretary of Commerce for Standards and Technology and Director 


\title{
Method for Measuring Axis Orthogonality in MEMS Accelerometers
}

\author{
Craig D. McGray, ${ }^{*}$ Yaqub Afridi ${ }^{\mathrm{a}}$, and Jon Geist ${ }^{\mathrm{a}}$ \\ ${ }^{a}$ Semiconductor and Dimensional Metrology Division, \\ National Institute of Standards and Technology, Gaithersburg, MD 20899
}

\begin{abstract}
A method is described for the computation of axis orthogonality errors in 3-axis accelerometers, based on the application of gravitational force at known angles. A precision twoaxis articulated gimbal system is used to control the angle at which the gravitational force acts on the accelerometer, while accelerometry data are collected at each applied angle. The resulting data are fit to a multivariate polynomial, and the coefficients of the linear terms are used to calculate the direction of each axis of the accelerometer and the angle between each axis pair.
\end{abstract}

${ }^{*}$ Corresponding author: C. D. McGray Tel: 301-975-4110. Fax: 301-975-6021.

100 Bureau Dr., MS 6830, Gaithersburg, MD 20899

E-mail address: craig.mcgray@nist.gov 


\section{Introduction}

Many modern commercially-available accelerometers report accelerations along three distinct axes so that both the direction and magnitude of an acceleration acting on the sensor can be determined. It is typically intended that these three measurement axes all be mutually orthogonal. Manufacturing defects will produce some amount of error in the orthogonality of these axes, which will in turn effect the error in the acceleration vector calculated from the accelerometer's output. It is hypothesized that axis orthogonality errors may be particularly stable components of the total error of an accelerometer, and that they may therefore yield to correction based on onetime calibrations.

To determine the orthogonality of the accelerometer axis pairs, the static response of each axis to gravity is measured as the accelerometer is rotated through the space of possible orientations on a precision gimbal mount. Once the response function has been measured, the direction of each sensor axis is determined relative to an imposed external coordinate frame. The three angles between these directions can then be easily calculated.

\section{Conventions, Definitions, and Assumptions}

We assume that the device under test will be an accelerometer having three scalar outputs corresponding to a right-handed set of axes that are all mutually orthogonal to within some small error angle. We will restrict our characterization of the device's performance to its response to a constant acceleration of $g$, where $g$ is the acceleration due to gravity. We assume a uniform gravitational field in the location where the experiment will be conducted. 
Without loss of generality, we impose an extrinsic right-handed coordinate system defined by an orthonormal basis, $B_{1}=\{\hat{x}, \hat{y}, \hat{z}\}$, in which $\hat{z}$ is parallel to, and opposite in sign to, the direction of gravity. We impose an intrinsic coordinate system defined by the orthonormal basis $B_{2}=\{\hat{i}, \hat{j}, \hat{k}\}$, which rotates with the accelerometer. $B_{2}$ is defined to equal $B_{1}$ when the accelerometer has first been fixed to the gimbal mount and the gimbal axes are set to zero rotation.

The static stimulus applied to an accelerometer or to an axis of an accelerometer is a vector corresponding to the magnitude and direction of the applied acceleration. (We will not treat vibration.) For static accelerometer tests, the stimulus will always equal $-g \hat{z}$.

The static response of any given axis of the accelerometer to a static stimulus is a scalar value that depends on the stimulus and on the orientation of the accelerometer. The static response of the accelerometer as a whole is a vector corresponding to the static responses of the three accelerometer axes.

For an ideal accelerometer, the response along a given axis with direction $\hat{u}$, to a static stimulus, $\vec{v}$, will be $R_{\hat{u}}(\vec{v})=s(\hat{u} \cdot \vec{v})+b$, where $s$ and $b$ are constants reflecting the sensitivity and bias of the accelerometer, respectively.

The static response of an axis of a real accelerometer is expected to approximate $s(\hat{u} \cdot \vec{v})+b$ but to deviate in ways that may include offset (zero-g bias), nonlinearity, and crosstalk. It can be approximated to arbitrary complexity by Maclaurin series expansion as:

$$
R_{\bar{u}}(\vec{v})=\sum_{\ell=0}^{\infty} \sum_{m=0}^{\infty} \sum_{n=0}^{\infty}\left(c_{\ell, m, n} i^{\ell} j^{m} k^{n}\right)
$$

where each $c_{\ell, m, n}$ is a constant and the coordinates of $\vec{v}$ in the intrinsic basis are $i, j$, and $k$. 
For example, a series expansion of Equation 1 containing only $0^{\text {th }}$ and $1^{\text {st }}$ order terms produces the expression for an ideal accelerometer defined above.

The noise in the static response of an accelerometer axis is the portion of the variation in the measured response over time that exhibits a wavelength that is lower than the measurement duration. The drift in the static response is the portion of the variation in the measured response over time that exhibits a wavelength that is greater than or equal to the measurement duration.

\section{The Three-Axis Accelerometer Static Response Transform}

The static response transform for all three axes of an ideal accelerometer can be expressed in matrix notation as follows:

$$
{ }^{1} R(\vec{v})=\left[\begin{array}{c}
a_{1} \\
a_{2} \\
a_{3}
\end{array}\right]=\vec{b}+S \vec{v}
$$

where $\vec{v}$ is a static stimulus with coordinates expressed with respect to the intrinsic basis $B_{2}=\{\hat{i}, \hat{j}, \hat{k}\}, a_{1}, a_{2}$, and $a_{3}$ are the response values of the three axes of the accelerometer, $S$ is

a $3 \times 3$ matrix representing the directions and sensitivities of the three axes, and $\vec{b}$ is a vector representing the biases of the three axes. Equation 2 corresponds to a first-order approximation generated from Equation 1. If the axes of the accelerometer were to align perfectly with the axes of the intrinsic basis, then $S$ would be diagonal.

Extension to a second-order approximation of the response transform (from Equation 1) yields:

$$
{ }^{2} R(\vec{v})=\left[\begin{array}{c}
a_{1} \\
a_{2} \\
a_{3}
\end{array}\right]=\vec{b}+S\left[\begin{array}{l}
i \\
j \\
k
\end{array}\right]+T\left[\begin{array}{c}
i^{2} \\
j^{2} \\
k^{2}
\end{array}\right]+U\left[\begin{array}{c}
i j \\
i k \\
j k
\end{array}\right]
$$


where $i, j$, and $k$ are the coordinates of $\vec{v}$ with respect to $B_{2}$. Higher-order approximations of the response transform can be generated from Equation 1 as needed.

\section{Accelerometer Axis Directions}

Regardless of which order of approximation of the static response transform is generated from Equation 1, the expression of the transform will include the linear matrix multiplier $S$. This matrix can be viewed as a change of basis from the intrinsic basis $B_{2}=\{\hat{i}, \hat{j}, \hat{k}\}$ to the accelerometer basis, $A=\left\{\vec{a}_{1}, \vec{a}_{2}, \vec{a}_{3}\right\}$, thereby defining the axes of the accelerometer. To calculate the coordinates of the basis vectors of $A$ with respect to $B_{2}$, we solve:

$$
[\vec{v}]_{A}=S[\vec{v}]_{B_{2}} \text { for each }[\vec{v}]_{A} \in\left\{\left[\begin{array}{l}
1 \\
0 \\
0
\end{array}\right],\left[\begin{array}{l}
0 \\
1 \\
0
\end{array}\right],\left[\begin{array}{l}
0 \\
0 \\
1
\end{array}\right]\right\}
$$

EQUATION 4

Yielding the vectors $\left\{\left[\vec{a}_{1}\right]_{B_{2}},\left[\vec{a}_{2}\right]_{B_{2}},\left[\vec{a}_{3}\right]_{B_{2}}\right\}$. The angle $\theta_{\ell m}$ between each pair of accelerometer basis vectors $\vec{a}_{\ell}$ and $\vec{a}_{m}$ can then be calculated by:

$$
\left[\vec{a}_{\ell}\right]_{B_{2}} \cdot\left[\vec{a}_{m}\right]_{B_{2}}=\left\|\left[\vec{a}_{\ell}\right]_{B_{2}}\right\|\left[\mid \vec{a}_{m}\right]_{B_{2}} \| \cos \theta_{\ell, m}
$$

EQUATION 5

The elements of the vector $\vec{b}$, the matrix $S$, and the higher-order matrices (if used) can be determined by fitting Equation 2 (or Equation 3, or a higher-order approximation) to the response generated by the accelerometer upon applying a series of static stimuli with known magnitude and direction with respect to the intrinsic basis. These known stimuli can be applied by gravity if the change-of-basis transformation between the extrinsic and intrinsic bases is known.

\section{Propagation of Uncertainty}


Given known uncertainties on the elements of $S$, the combined variance of each $\theta_{\ell, m}$ can be determined from the Law of Propagation of Uncertainty [1] (assuming statistical independence of the inputs) as follows:

$$
u_{c}^{2}(\theta)=\sum_{m=1}^{3} \sum_{n=1}^{3}\left(\frac{\partial \theta}{\partial S_{m, n}}\right)^{2} u^{2}\left(S_{m, n}\right)
$$

where $u\left(S_{m, n}\right)$ is the uncertainty of the element in the $m^{\text {th }}$ row and $n^{\text {th }}$ column of $S$, and where the partial derivatives can be calculated from Equations 4 and 5 .

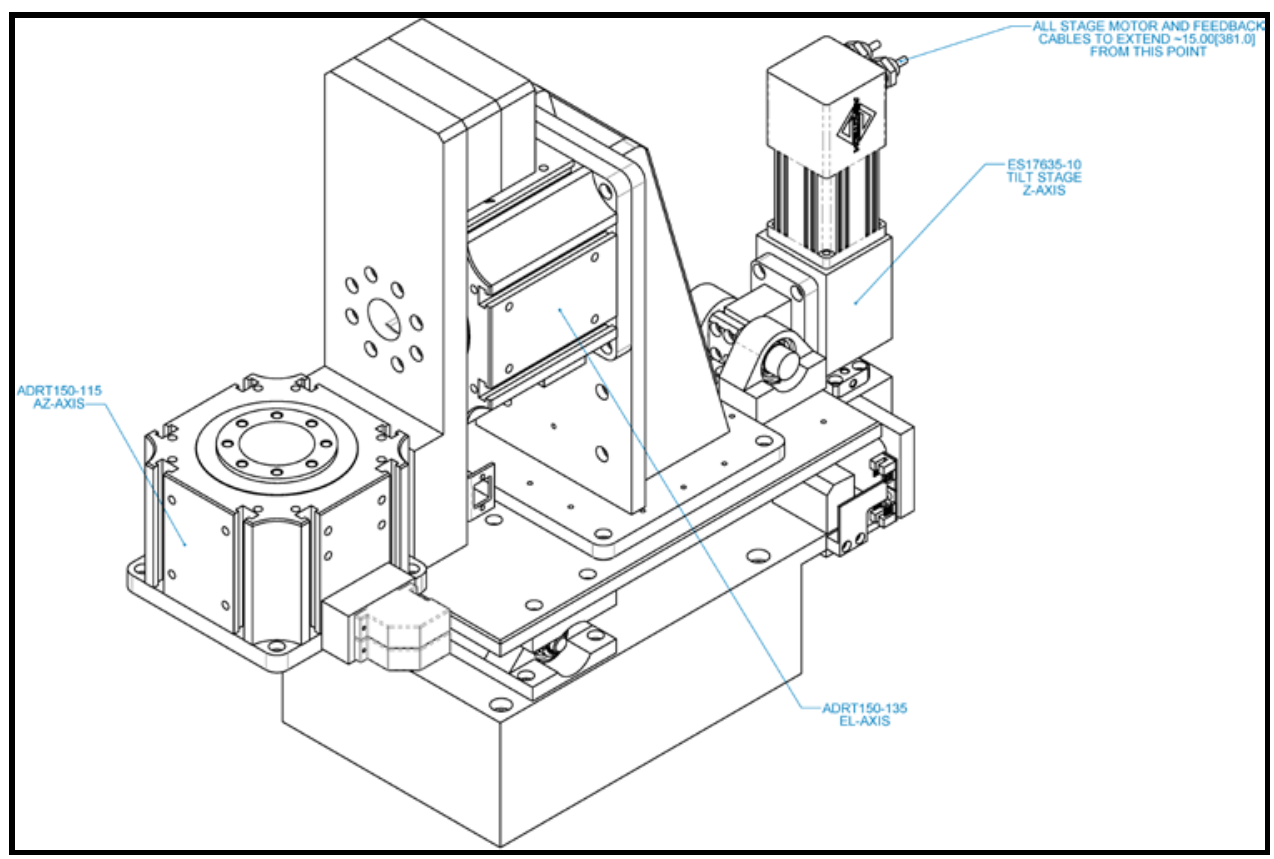

Figure 1 - Diagram of the articulated gimbal system.

\section{Gimbal System Design}

The change-of-basis transformation between the extrinsic and intrinsic bases can be determined with an instrumented gimbal system that rotates the accelerometer with two orthogonal degrees of freedom. The gimbal system utilized for these tests is configured in a rollover-elevation configuration with $360^{\circ}$ of travel in each axis so that the gravitational force can be 
applied to each axis of the accelerometer from all possible angles. Positioning accuracy of each axis of the gimbal system is better than 18 arcsec, with orthogonality of the two axes better than $0.05^{\circ}$. A third tilt axis with a range of $\pm 2^{\circ}$ is included to allow alignment of the elevation axis with respect to the direction of the local gravitational field. A diagram of the gimbal system appears in Figure 1.

\section{Gimbal System Calibration}

Calibration of the articulated axes of the gimbal system is performed by rotating a calibrated alignment cube through the positions of the gimbal while measuring the rotation of the alignment cube using a pair of autocollimators. Measuring concurrently with two autocollimators allows the global rotation error to be assessed. With a cubic alignment calibration artifact, only $90^{\circ}$ rotations of the gimbal can be accommodated. Each axis of the instrument is therefore tested across $90^{\circ}$ rotations beginning at each of $\mathrm{N}$ equally-spaced orientations, while the other axis is held fixed in place.



Figure 2 - Sub-gimbal assembly for calibrating the articulated gimbal. 
To facilitate the calibration process, the alignment cube is mounted on a manual two-axis gimbal assembly which in turn is mounted on the roll axis of the articulated gimbal as shown in Figure 2. The two autocollimators are aligned to adjacent faces of the alignment cube. One axis of the articulated gimbal is rotated by exactly $90^{\circ}$, the change in alignment of each autocollimator relative to the cube is recorded, and the axis is returned to its starting position plus 360/ $N$ degrees. The misalignment between the cube and the autocollimators resulting from this motion may exceed the measuring range of the autocollimators. If so, then the axes of the manual sub-gimbal assembly are adjusted to compensate. The articulated gimbal is then again rotated by exactly $90^{\circ}$. This process is repeated until all $\mathrm{N}$ rotations have been measured.

The differences at each step, as measured by autocollimator, between the true rotation of the gimbal actuator and the nominal (i.e. commanded) rotation can be used not only to check that the gimbal is within its specified accuracy, but also to refine or correct the accuracy of the gimbal. Let $\theta$ be the nominal orientation of the gimbal actuator relative to its home position, and let $\varphi$ be the true orientation. We can say that $\varphi(\theta)=\theta+\varepsilon(\theta)$, where $\varepsilon$ is the positioning error. The $i^{\text {th }}$ autocollimator measurement can then be expressed in terms of the nominal orientation as follows:

$$
m_{i}=E\left(\frac{2 \pi i}{N}\right)=\varepsilon\left(\frac{2 \pi i}{N}+\frac{\pi}{2}\right)-\varepsilon\left(\frac{2 \pi i}{N}\right)
$$

If the positioning error, $\varepsilon(\theta)$, is modeled as a uniform $k$-segment cubic spline, where $\mathrm{k}$ is a multiple of 4 , then $E(\theta)$ will also be a uniform $k$-segment cubic spline. The autocollimator measurements $m_{1} \ldots m_{N}$ can be fit to a uniform $k$-segment cubic spline to determine the function $E(\theta)$. The positioning error function, $\varepsilon(\theta)$, can then be found by solving Equation 8 for the spline coefficients of $\varepsilon(\theta)$ : 


$$
E(\theta)=\varepsilon\left(\theta+\frac{\pi}{2}\right)-\varepsilon(\theta)
$$

A similar analysis can be performed to calculate the true cross-axis orientation, $\varphi_{+}(\theta)=0+\varepsilon_{+}(\theta)$, where $\varepsilon_{+}(\theta)$ is the cross-axis positioning error function.

\section{Gimbal Initialization}

If the two articulated gimbal axes are orthogonal to one another and the elevation axis is orthogonal to the direction of the local gravitational field, then force can be applied to the accelerometer along any direction relative to the accelerometer axes simply by selecting the appropriate rotations of the two articulated gimbal axes. To ensure orthogonality to gravity, the gimbal may be initialized prior to use.

To initialize the gimbal, the elevation axis of the gimbal is first moved to its home position, in which the gimbal will appear as shown in Figure 1. The roll axis in this configuration is nominally parallel to gravity, but may be offset by some small angle due to non-planarity of components of the gimbal or due to tilt in the surface on which the gimbal is situated. The roll axis is then set to rotate continuously, and the output of the accelerometer is monitored. If the roll axis is parallel to gravity, then the force vector acting on the accelerometer will hold constant as the roll axis rotates. If not, then the accelerometer output will vary cyclically with each revolution. The gimbal operator then adjusts the articulated elevation axis and the manual tilt axis until the variation in the accelerometer output is nulled.

\section{Measurement of the Accelerometer Response Transform}

With an accelerometer mounted on the roll axis of the calibrated gimbal, and with the gimbal axes initialized, measurement of the accelerometer response transform can be performed. A set of coordinate pairs $(\theta, \psi)$ is selected, with $\theta$ corresponding to the elevation axis, and $\psi$ 
corresponding to the roll axis. While a number of methods exist to select coordinate pairs so that they are distributed approximately equally throughout the spherical parameter space, one simple way is as follows. If approximately $N$ coordinate pairs are desired, let $I$ be the set of integers between $-\sqrt{N} / 2$ and $\sqrt{N} / 2$. For each $I_{m} \in I$, let $\theta_{m}=\left|\arccos \left(2 I_{m} / \sqrt{N}\right)\right|$, and let $\psi_{m}=\left(I_{m}+\sqrt{N} / 2\right) \frac{\pi}{\sqrt{N}}$. With the gimbal axes positioned at each coordinate pair, the output values of the accelerometer are collected for a constant time, $t$, and averaged to reduce the effect of noise.

Each point in the resulting data set is therefore a 5-tuple of the form $\left\{\theta, \psi, a_{1}, a_{2}, a_{3}\right\}$, where $\theta$ is the orientation of the elevation axis, $\psi$ is the orientation of the roll axis, and $\left\{a_{1}, a_{2}, a_{3}\right\}$ are the values reported by the three axes of the accelerometer. The elevation and roll axis orientations can be used to change the basis of the static stimulus, $g \hat{z}$, from the extrinsic basis, $B_{1}$ to the intrinsic basis, $B_{2}$. This is achieved simply by multiplying the stimulus vector by the following rotation matrix:

$$
[g \hat{z}]_{B_{2}}=\left[\begin{array}{ccc}
\cos \theta \cos \psi & \sin \psi & -\sin \theta \cos \psi \\
-\cos \theta \sin \psi & \cos \psi & \sin \theta \sin \psi \\
\sin \theta & 0 & \cos \theta
\end{array}\right][g \hat{z}]_{B_{1}}
$$

Having performed this rotation, each point in the data set is now a 6-tuple of the form $\left\{i, j, k, a_{1}, a_{2}, a_{3}\right\}$, where $i, j$, and $k$ are the coordinates of the stimulus with respect to the intrinsic basis, $B_{2}$. Once in this format, the data can be fit to Equation 2, Equation 3, or a more complex model function derived from Equation 1. The matrix multiplier, $S$, can be extracted from the linear term of the model function, allowing the components of the accelerometer basis 
$A=\left\{\left[\vec{a}_{1}\right]_{B_{2}},\left[\vec{a}_{2}\right]_{B_{2}},\left[\vec{a}_{3}\right]_{B_{2}}\right\}$ to be calculated according to Equation 4 . Once the accelerometer basis is known, the accelerometer axis angles can be calculated according to Equation 5.

\section{REFERENCES}

[1] W. G. ISO Technical Advisory Group 4, "Guide to the expression of uncertainty in measurement," ed: International Standards Organization, 1995.

[2] C. D. McGray, R. A. Allen, M. Cangemi, and J. Geist, "Rectangular scale-similar etch pits in monocrystalline diamond," Diamond and Related Materials, vol. 20, Nov. 2011. 